\title{
The SAGE Encyclopedia of Research Methods
}

\section{Entry Title: Reflexivity}

Authors: [list all authors, add more row if needed]

$1 \quad$ Name Paul Atkinson

Affiliation, country Cardiff University, United Kingdom

Lead author email AtkinsonPA@Cardiff.ac.uk

SAGE Author ID [office use only]

2 Name Emilie Morwenna Whitaker

Affiliation, country University of Salford

SAGE Author ID [office use only]

Discipline(s) Sociology [D1]

Method Group Qualitative

Entry Category Key Concepts

Entry Size (approximate word count) $\quad 5,000$

URL http://methods.sagepub.com/foundations/

\section{Reflexivity}

Central to reflexivity is an awareness that the researcher and the object of study exist in a mutual relationship with one another. Thus, reflexivity calls for attention to how thinking comes to be, how it is shaped by pre-existing knowledge, and how research claims are made. The topic of reflexivity is pervasive in the methodological literature of the social sciences. It is an issue for the social sciences in general, but it has particular significance for ethnographic and other qualitative research (Davies, 2008). There is no single definition of reflexivity: it has multiple meanings and connotations (Babcock, 1980). . Moreover, it is apparent that some usages of 'reflexivity' actually overlook its core significance. The aim of this entry is to clarify some of the main issues raised by reflexivity. This entry begins by discussing reflexivity in social research. It then examines several types of reflexivity, including epistemic, disciplinary, methodological, textual, and positional reflexivity. The entry concludes by briefly contrasting reflexivity and reflection. 


\section{Reflexivity in Social Research}

Reflexivity runs through the research endeavour. Its recognition enjoins: considering the bases of theoretical assumptions; being open to revision in the light of observations; examining methodological assumptions; being conscious of the disciplinary lenses that inform current thinking. Reflexivity is not just 'reflection' at a personal level, although it implies reflective practice: reflection about the self of the researcher-biases, preferences, biography and practice-is the basis for sound and ethical research but it is not the whole story about reflexivity. In an early intervention, Barbara Myerhoff and Jay Ruby (1982) suggested that 'Reflexive, as we use it, describes the capacity of any system of signification to turn back upon itself, to make itself its own object by referring to itself: subject and object fuse' (p. 2). In the intervening years, this has developed into a more complex array of epistemological and methodological issues, which are explored in this entry.

As a high level of abstraction, reflexivity has been used as a major theme by a number of leading social theorists. In general, they use it to refer to the specific property of social science: that social actors are themselves actively engaged in construing their own everyday lives and the social world around them. Consequently, the kinds of analysis that social scientists perform can feed back directly into the social lives that they study. Scholarly, analytic concepts can thus become incorporated into first-order, mundane understandings. There is, therefore, the possibility for circularity between the sociological understanding of the social and the incorporation of that understanding into their self-understanding-a form of 'hermeneutic circle'. The social and the sociological are thus mutually constituting. This is the thrust of 'reflexivity' as employed by theorists such as Anthony Giddens. Such high-level generalisation is not, however, productive in making sense of the practice of social research, which is the main purpose of this entry.

Reflexivity in social research is not an option. It is a fundamental and inescapable feature of all research, in the natural and social sciences alike. Reflexivity refers to the inescapable fact that the research act, however conducted, in part constructs or defines the phenomenon that is the object of that research. Any act of observation or measurement is a form of intervention, and to that extent all research is reactive. There is no perfectly neutral vantage-point, and no transparent medium of description, that exempt the observer from reactivity. This is by no means confined to the social sciences; it is, a fundamental feature of all science. Any measurement or observation inevitably frames the phenomena under investigation. This is especially pertinent in contemporary physics. At the quantum level, the very act of observation has a consequence in defining the state of the waves or particles that are being observed.

The conceptual task is complicated by some claims surrounding reflexivity that are poorly grounded in the epistemological foundations of social science. Rather than being understood as a matter of collective research practice, over-simplified uses of 
the term imply individual choice, and even personal virtue. In this oversimplification, reflexivity is portrayed as a matter of reflection and interpersonal sensitivity on the part of the researcher, suggesting that reflexivity (a condition of all research) is equivalent to reflection or reflective practice (an individual research virtue). As noted by Karl Maton (2003), it certainly should not be equated with a narcissistic obsession with the researcher's own self, feelings and reactions.

In order to clarify the issues, it is important to recognise that there are several different kinds or 'levels' of reflexivity. They are each dealt with separately here. Ultimately they interact with one another in complex ways. Methods of measurement-however defined-interact with the social or natural world, and in the course of measurement they construct the phenomena under investigation. There is no escape from the fact that any act of observation or description plays a part in the scientific construction of the very phenomena under investigation. This is not the same as a radically constructivist perspective that denies or minimises the existence of a 'real' world. But it does mean that there is no purely transparent or neutral way of engaging with and describing that reality.

\section{Epistemic Reflexivity}

The sociology of knowledge recognises that knowledge is perspectival, or relational. Karl Mannheim's sociology of knowledge, for instance, recognises that 'the social qualities of the knower shape the characteristic of his/her thought, not only with regard to the genesis of ideas, but also concerning the form and contents as well as the formulation and intensity of experience' (Mendel, 2006, p. 31). That does not mean that reflexivity prevents any sense of a material or social 'reality'. It is not a reflection of a purely relativist position in that sense. But it does highlight the extent to which any knowledge of it is situated, partial, and subject to revision. Equally, it does not mean that one can or should abandon all attempts at systematic, disciplined inquiry. It does, however, enjoin sustained, analytic attention to the nature and consequences of reflexivity.

The general perspective of epistemic reflexivity is closely associated with Pierre Bourdieu (e.g., Bourdieu, 1990; Bourdieu \& Wacquant, 1992). Reflexivity, in Bourdieu's terms, rests on three fundamental issues. First, it is an issue of the 'intellectual unconscious' that is reflected in research practices. Second, it is a collective matter, not a problem of individual consciousness. Third, his treatment of reflexivity is not intended to undermine the objectivity of sociology (and he deals only with sociology), but rather to enhance it. The social orientation of the investigator inevitably impinges on her or his scientific imagination. But it is not solely, or even primarily, a matter of personal identity. Reflexivity is a collective matter, grounded in what Bourdieu calls the 'intellectual field'. One's general intellectual orientationphilosophical, epistemological or theoretical-inevitably frames how research questions are framed. 
In other words, intellectual fields-that can be glossed as disciplines or subdisciplines-inevitably shape the framing of research questions. At a gross level that seems self-evident, what are superficially 'the same' phenomena will be identified very differently by sociology, anthropology, psychology and economics. Each discipline is based on its distinctive models of 'the social' and of the 'social actor'. More subtly, the same holds within a major discipline such as sociology. A perspective such as symbolic interactionism will foreshadow a very different array of phenomena than, say, rational-choice theory, or structural functionalism. Disciplines are not static. They change over time. Some of that change reflects cumulative knowledge, some of it is driven more by fashionable trains of thought, while some reflects the dominance of key thinkers and concepts. Consequently, the topics of research differ over time as well as between disciplines or schools of thought. Bourdieu himself regarded his entire sociological work as a reflexive scrutiny of the grounds of his own sociology: his analysis of French academics and their formation; the sociology of education; the social distribution of cultural resources. Like other treatments at a high level of abstraction, however, Bourdieu's version of reflexivity is too generalised to illuminate actual practices of social research. His emphasis is also on the neutralisation of bias and the strengthening of sociology's objectivity. But the pervasiveness of reflexivity suggests that anxieties concerning 'bias' are misplaced. One cannot eliminate the reflexive nature of discipline and method. One can, however, as Bourdieu himself attempts, treat the practices of social research as an object of inquiry at the same time as undertaking social-scientific research. It is a matter of collective transparency and accountability rather than the elimination of a reflexivity effect.

Epistemic reflexivity has a number of components, or levels, that need to be identified, since it is not susceptible to reduction to simple formulations and responses. The following sections outline some of the key elements that logically follow from a general understanding of epistemic reflexivity. They are not mutually exclusive. They interact in multiple ways. They are distinguished here for the purpose of clarity and for ease of discussion.

\section{Disciplinary Reflexivity}

Irrespective of whether social scientists identify themselves closely with one discipline, or whether they embrace a more fluidly interdisciplinary stance, intellectual traditions bear directly on the nature of research and the phenomena that are identified. In other words, disciplines set the possibilities of research. They help to define what is worth studying, what counts as worthwhile or newsworthy subjectmatter, what is worth taking seriously, and how to identify it. Such disciplinary reflexivity is simultaneously productive and constraining. It is productive, in that it can suggest fruitful lines of inquiry, and furnish the means to pursue them. It can provide the researcher with templates and exemplars. At the same time, it can constrain research, precisely because those lines of inquiry can implicitly exclude or marginalise other phenomena, rendering them 'unthinkable'. Disciplinary framing is 
often tacit, reflecting, as it does, the shared taken-for-granted assumptions of an intellectual field.

To that extent, the reflexivity of disciplinary knowledge and tradition can be likened to the notion of a collective thought-style (Fleck, 1937/1979) or a scientific paradigm (Kuhn, 1962). The paradigm is not just a preference for one theory over another at a given point in time. It is a package of key ideas, key works, accepted methods, leading figures and role models, and classic studies. A paradigm frames simultaneously what to study, the appropriate methods to identify the most relevant phenomena, and what the expected outcomes should be.

Although this entry refers to 'disciplinary reflexivity', this does not mean that any given discipline will display one homogeneous array of guiding ideas. The outcomes of disciplinary reflexivity are not immutable either. Indeed, an understanding of the reflexive framing of research needs to be sensitive to the internal differentiations of the discipline. Disciplinary reflexivity reflects changing research foci in the parent discipline. For instance, feminist and queer standpoints reflexively re-frame the proper subject-matter of the social sciences (Harding, 2004; Sullivan, 2003). So too does the emergence of critical race theory (Delgardo \& Stefanovic, 2017). There is, therefore, a dialectical relationship whereby observed phenomena in the field and key disciplinary ideas interact with one another.

As Bourdieu pertinently observes, the intellectual field not only provides paradigmlike frames for the design and conduct of research, it also furnishes the critical audience for that research. The researcher's 'peers' therefore evaluate the worth of the research against the collectively shared-often implicit—criteria that inform the choice and identification of researchable phenomena in the first place. The intellectual field sets the rules of the game.

\section{Methodological Reflexivity}

Reflexivity implies that research methods frame the phenomena to be studied. The collective examination of methodology and standpoint parallels the recurrent interest in what is sometimes called methodography. That is, a close examination of how the methods of the social sciences are deployed, and how they shape their objects. In many ways that is the overall tenor of Aaron Cicourel's landmark contribution on measurement in sociology (Cicourel, 1966). The general message was that social sociologists were far too reliant on measurements (not just quantitative) that imposed unexamined assumptions in categorising phenomena. The argument can be applied to all methods. Methodography satisfies-in part-Bourdieu's injunction that academics should include their own practices within their objectifying scrutiny.

Since reflexivity refers to the inescapable effects of any research intervention, it means that the research methods used will reflexively shape the kinds of 
phenomena that are identified, classified and measured. The core of ethnographic research, participant observation, is perhaps the most self-evidently reactive method, depending as it does on the direct engagement of the researcher with her or his research hosts. This is not just a matter of the researcher's presence in the field. It rests on the fact that the phenomena that are describable, and the events that are reportable, are largely the outcomes of the encounters that are possible and achievable in the field. Indeed, the 'field' of fieldwork is the outcome of successive negotiations between the ethnographer and groups or individuals.

Reflexivity of method is not unique to ethnography, however. Extended and in-depth interviewing is a common feature of contemporary social science, and it is undertaken in many ways. All interviewing presupposes particular relationships between the researcher and the interviewee. Moreover, the interview can itself inscribe particular expectations of constructions of what kind of 'data' will be forthcoming. Such presuppositions can also project kinds of social actors-speaking subjects who will display selves and identities in an appropriate manner. As Charlotte Aull Davies (2008) points out, the interview is not a culturally universal phenomenon. Moreover, even within Western culture, the interview is a highly variable encounter. It can range from a simply vox pop interview with the man-orwoman-in-the-street, to feature-length interviews conducted by writers with celebrities and public intellectuals. Paul Atkinson and David Silverman (1997) suggested that the widespread reliance on interviewing in qualitative (including ethnographic) research is a symptom of what they called the 'interview society'. The culture of the interview, they suggested, is preoccupied by a search for how respondents 'feel' about their personal experiences. Such interviewing therefore presupposes the revelatory or confessional outcome of the research.

Methodological reflexivity is not confined to qualitative research. The quantitative disciplines also imply a close interdependence between the choice of method and assumptions about the phenomena they describe. The discipline of economics has, for much of its existence, been predicated on disciplinary assumptions about its subject-matter: the nature of the social actor as a decision-making being, or the nature of markets. The methods of economics construct social actors and economic behaviour in distinctive ways. Likewise, as Cicourel discussed at length, the methods of survey research depend on particular kinds of measurement and classification that in turn construct human subjects as particular kinds, that 'fit' predispositions, motives and behaviour into pre-determined categories. This is not based on a rejection of enumeration and quantitative methods (as some simplistic distinctions between quantitative and qualitative methods might seem to imply), but is just one among many ways in which methods constrain as well as generate social research and its findings. A complementary perspective, from a somewhat different disciplinary stance, is suggested by Richard Biernacki (2012) who addresses the contentious nature of coding data in cultural and social research, which he identifies as part of the 'ritual' of research in such disciplines. 
Methodological reflexivity also means that simple models of triangulation do not work. Different methods do not simply provide complementary sources of information about the same phenomena. Methods configure phenomena differently, and constitute different phenomena. In the most rigorous of terms, the outcomes of different methods may be incommensurable. Even a pragmatic view of different methods and strategies implies that data of different sorts must be treated with a rigour that respects their intrinsic properties. Different kinds of data are not simply additive: for instance, Atkinson and Amanda Coffey (2002), discussed the vexed question of the appropriate relationship between participant observation and interviewing. Although the two strategies are often used in conjunction, their relationship is not straightforward, and they cannot be used to confirm or validate one another on similar issues. For the same reason, enthusiasm for mixed methods needs to be treated with caution, as 'mixing' methods can run into the same problems as triangulation. Methodological reflexivity is one of the most fundamental issues in social research.

\section{Textual Reflexivity}

One of the most significant aspects of reflexivity in the social sciences resides in the textual means of representing social worlds, social scenes and social actors. This is in turn a manifestation of the essential reflexivity of accounts: accounts construct the phenomena that they describe in the course of their descriptions. This does not mean that accounts are completely arbitrary, or that they have no referential value. It does means that language has a constitutive function: there is no neutral or transparent medium of expression.

Again, textual reflexivity is a pervasive feature of research. It is not confined to the social sciences, although it receives explicit attention among certain social disciplines, anthropology and sociology in particular. A style of investigation and critique, known as the 'rhetoric of inquiry program' (Nelson, Megill \& McCloskey, 1987), has documented textual reflexivity across a wide range of subjects. For instance: the modern scientific paper obeys widely shared textual conventions, and those conventions frame what counts as proper scientific reporting; economics has its distinctive narrative and textual characteristics; narrative history has its distinctive genres; sociology deploys characteristic literary tropes. Disciplinary fields and specialisations prescribe textual formats, from the journal paper in the natural sciences to the ethnographic monograph in anthropology. Textual forms embody distinctive styles of thinking and paradigms. Textual reflexivity has been a major, recurrent theme in the literature on ethnography, both in anthropology and in sociology.

There are several strands to the relevant literature. They include publications that focus primarily on a critique of anthropology's conventions, and others that are more 
concerned with a dissection of ethnography's textual conventions. Obviously, the two perspectives converge at key points. Of the critical texts, the most prominent and influential was the collection Writing Culture (Clifford \& Marcus, 1986). It was argued that the textual style of the traditional anthropological monograph inscribed a characteristic stance on the part of the anthropologist. Anthropological monographs characteristically inscribed an implicit image of the ethnographer-author as an omniscient observer, who was simultaneously absent from the text. From within the sociological tradition, John Van Maanen (1988) identified distinctive genres of ethnographic reportage, and Atkinson (1990) described a variety of textual strategies that are deployed in ethnographic writing to persuade readers of the veracity of the published account.

\section{Positional Reflexivity}

Positional reflexivity does not reduce the issue to matters of individualised biography. It does, however, recognise that the researcher's identity has implications for all levels of reflexivity. Positionality here implies that issues of gender, ethnicity and status are all implicated in the research process. But such positionality should not be understood in mechanistic terms. A researcher's positionality-for example, ethnicity or sexual orientation-may reflexively interact with her or his disciplinary commitments in leading the researcher towards framing researchable phenomena and following particular methods.

Positionality may lead researchers towards feminist standpoint perspectives, or queer theory, or critical race theory. Equally, however, such reflexive framing of research should not be regarded as deterministic-any more than the other dimensions of reflexivity. It is axiomatic for social scientists to regard identities as fluid. It would, therefore, be a failure of intellectual nerve to assume that one's gender, ethnicity, nationality or sexual orientation would exert a totally determining effect. Biographical reflexivity is a particular issue, given the distinctive nature of social research, and of ethnography in particular. The gaze of participant observation passes in two directions, in the mutual interrogation of the ethnographer and her or his hosts. This goes beyond the reflexivity of most natural sciences, where the phenomena are framed by the observer (scientist) but that observer is not subject to the reverse gaze. The fieldwork of ethnography is predicated on interactions, encounters and conversations. It is by definition dialogic, at least in that limited sense. Consequently, biographical reflexivity is partly determined not just by the ethnographer's ascribed characteristics, but also by the host's perceptions and judgments of her or him. The ethnographer's self-presentation and the reciprocal moral evaluations influence the nature and scope of data collection, as well as pervading the ethnographer's interpretative frameworks. But this is by no means confined to ethnographic fieldwork. All social research needs to acknowledge the fundamental difference between the social and natural sciences: everyday social 
actors are themselves actively engaged in making sense of their own and others' actions. And that includes the research process itself: reflexivity encompasses the mutual understanding of researchers and the actors being researched.

\section{Reflexivity and Reflection}

Myerhoff and Ruby (1982) observed that reflexivity implies reflection, but the two are not the same: "Reflective" is a related but distinguishable term, referring also to the kind of thinking about ourselves, showing ourselves to ourselves, but without the requirement of explicit awareness of the implications of our display' (p. 3). Reflexivity implies professional reflection. A recognition of the reflexive nature of research enjoins all researchers-social scientists especially - to pay due attention to the multiple levels of reflexivity, and to the extent to which theories, methods and texts frame the research and its outcomes. But reflexivity is not synonymous with reflection. Reflecting on research, and on one's own engagement with research and its phenomena, is something that scholars can and should consciously do. Reflexivity, on the other hand, is an inescapable part of all research, whether or not it is explicitly recognised. Reflexivity therefore should not be reduced to a purely individual act. In the contemporary climate, it is commonplace to find authors claiming to have undertaken 'reflexive research' as a matter of methodological virtue, whereby the researcher/author apparently exposes her or his autobiographical origins and predispositions. When this is treated as a personal virtue, insufficient attention is paid to the epistemic and collective aspects of research reflexivity. It is not recognised that the reflexivity of research is what makes it possible in the first place: disciplinary knowledge, methodological means, forms of representation. It provides the essential character of research and is not just the cause of bias or error that can be eliminated.

Personal, autobiographical reflection undoubtedly has its place in the conduct of research. Reflection promotes honesty and transparency in the research process. It becomes dysfunctional, however, if it only collapses all of the intricacies of reflexivity into an individualised act, sometimes amounting to introspection. There remains, therefore, the need to distinguish reflexivity from reflection, even though reflective practice is one response to an awareness of the reflexivity of research. But such reflection—and consequent practice-must be based on a thorough understanding of the issues discussed in this entry, not just on a personal self-examination. Epistemic reflexivity should not be confused with personal reflection. All of the aspects of research reflexivity need not be collapsed into a purely single and personal dimension.

Equally, therefore, reflexivity does not equate to a personal confessional. Social scientists, notably sociological and ethnographic ethnographers, have developed the genre of confessional 'tales of the field' (Van Maanen, 1988). The separation of such 
confessionals from the 'real work' of the published monograph or research paper appears to have minimised research reflexivity, as it left the main analysis relatively untroubled by the personalised accounts of the researchers. As reflexivity has become an increasingly explicit aspect of the research process, however, the personal aspects of the research process have become increasingly intertwined with the reporting of research itself. While that personalises the research process and enhances its transparency, it should not be equated with reflexivity in all its forms. As already noted, one cannot choose to 'be' reflexive, since all research is reflexive. One can choose to be reflective, and to render the research more open. Such a choice can partly address the critique of social-science publishing that constructs the (largely invisible) author as an omniscient and disembodied figure. The absent author is transformed into a presence, both 'in the field' and 'in the text'. There is the accompanying danger that such reflection (claimed as reflexivity) becomes foregrounded to the point of obscuring the purpose of research itself. In extreme forms of autoethnography, the researcher-author becomes the primary focus of the text. In such cases, the full implications of reflexivity in research become blurred it not invisible.

\section{FURTHER READING}

Alvesson, M. and Sköldberg, K. (2018) Reflexive Methodology: New Vistas for Qualitative Research. 3rd edition. London: SAGE.

Aunger, R. (2004) Reflexive Ethnographic Science. Walnut Creek CA: AltaMira.

May, T. and Perry, B. (2017) Reflexivity: The Essential Guide. London: SAGE.

Ruby, J. (ed.) (1982) A Crack in the Mirror: Reflexive Perspectives in Anthropology. Philadelphia: University of Pennsylvania Press.

https://doi.org/10.9783/9781512806434

Sprague, J. (2016) Feminist Methodologies for Critical Researchers, 2nd edition. Lanham MD: Rowman and Littlefield.

Steier, F. (ed.) (1991) Research and Reflexivity. London: Sage.

\section{REFERENCES}

Atkinson, P.A. (1990) The Ethnographic Imagination: Textual Constructions of Reality. London: Routledge.

Atkinson, P. and Coffey, A. (2002) Revisiting the relationship between participant observation and interviewing', in J.F. Gubrium and J. A. Holstein (eds) Handbook of Interview Research. Thousand Oaks CA:SAGE 
Atkinson, P. and Silverman, D. (1997) Kundera's Immortality: The interview society and the invention of the self, Qualitative Inquiry, 3: 304-325.

https://doi.org/10.1177/107780049700300304

Babcock, B.A. (1980) Reflexivity: definitions and discriminations, Semiotica, 30,1/2: 1-14. https://doi.org/10.1515/semi.1980.30.1-2.1

Biernacki, R. (2012) Reinventing Evidence in Social Inquiry. London: Palgrave. https://doi.org/10.1057/9781137007285

Bourdieu, P. (1990) Other Words: Essays Towards a Reflexive Sociology. Stanford CA: Stanford University Press.

Bourdieu, P. and Wacquant, L. (1992) An Invitation to Reflexive Sociology. Cambridge: Polity

Cicourel, A. V. (1964) Method and Measurement in Sociology. New York: The Free Press.

Clifford, J. and Marcus, G.E (eds) (1996) Writing Culture, Berkeley, CA. University of California Press.

Davies, C.A. (2008) Reflexive Ethnography: A Guide to Researching Selves and Others. 2nd edition. London: Routledge.

Delgardo, R. and Stefanovic, J. (2017) Critical Race Theory, 3rd edition. New York: New York University Press.

Fleck, L. (1937/1979) The Genesis and Development of a Scientific Fact. Chicago: University of Chicago Press.

Harding, S. (ed.) (2004) The Feminist Standpoint Reader. New York: Routledge.

Kuhn, T. S. (1962) The Structure of Scientific Revolutions. Chicago: University of Chicago Press.

Maton, K. (2003) Pierre Bourdieu and the epistemic conditions of social scientific knowledge, Space and Culture, 6, 1: 52-65.

https://doi.org/10.1177/1206331202238962

Mendel, I. (2006) Mannheim's free-floating intelligentsia: The role of closeness and distance in the analysis of society, Studies in Social and Political Thought, $12: 30-52$.

Myerhoff, B. and Ruby, J. (1982) Introduction, in Ruby, J. (ed.) A Crack in the Mirror: Reflexive Perspectives in Anthropology. Philadelphia: University of Pennsylvania Press, pp. 1-36 https://doi.org/10.9783/9781512806434-003

J.S. Nelson, A. Megill and D.N. McCloskey (eds). (1987). The Rhetoric of the Human Sciences. Madison: University of Wisconsin Press. 
Sullivan, N. (2003) A Critical Introduction to Queer Theory. New York: New York University Press.

Van Maanen, J. (1988) Tales of the Field. Chicago: University of Chica 